\title{
Superior surface hardening by cyclic-phase-change-diffusion (CPCD): Supersaturation of hard boride in titanium subsurface layer
}

\author{
B. Sarma and K. S. Ravi Chandran ${ }^{1}$ \\ Department of Metallurgical Engineering, University of Utah \\ 135 South 1460 East Rm. 412 Salt Lake City UT 84112
}

\begin{abstract}
This research demonstrates a new concept of surface hardening, termed "cyclic-phase-changediffusion (CPCD)," where cyclic phase transitions were induced at the surface by thermal cycling across the phase transition temperature along with diffusion of surface hardening atoms. Specifically, boron was diffused through the surface of titanium during thermal cycling across the $\alpha-\beta$ phase transition temperature. The CPCD method is shown to result in an unusually deeper $(\sim 125 \mu \mathrm{m})$ and richer boride layer, on titanium, compared that obtained by isothermal diffusion either at the equivalent temperature or at a higher temperature. This concept is quite promising for enhanced surface hardening of titanium.
\end{abstract}

\section{Keywords:}

Titanium boride, kinetics, thermal cycling, phase change, diffusion, titanium, boron

Thermal cycling as a microstructure modification method in metallurgy has led some interesting results in the past. Grange ${ }^{1}$ and others, ${ }^{2},{ }^{3}$ pioneered the use of thermal cycling to refine grain size in steels. Others ${ }^{4}$, showed the increased densification of metal powders when thermal cycles were imposed during sintering. More recently, it has been shown that thermal cycling enhances super-plastic deformation in titanium ${ }^{6}$ and shortens the time for completion of bainitic transformation $^{7}$ in steel.

Taking it to next level, the question of what happens when cyclical phase transformations are induced simultaneously during the diffusion of atoms, through the surface, is intriguing. It appears that this concept has not been investigated before. First, we examine here the nature of temperature distribution in the subsurface regions of a semi-infinite medium, induced by a

\footnotetext{
${ }^{1}$ Corresponding author, email: ravi.chandran@utah.edu
} 
harmonic variation in surface temperature. The differential equation governing the onedimensional heat transfer in a semi-infinite solid is

$\frac{\partial^{2} T}{\partial x^{2}}=\frac{1}{k} \frac{d T}{d t}$

Where $T$ is temperature, $k$ is thermal diffusivity, $x$ is distance from surface and $t$ is time. The solution for subsurface temperature distribution for the cosine surface temperature oscillation, $T=A \cos (\omega t)$ at $x=0$, is $\left(\right.$ Carslaw and $\left.\mathrm{Jaeger}^{8}\right)$ :

$T=T_{a} e^{-K x} \cos (\omega t-K x)$

where $\mathrm{T}_{\mathrm{a}}$ is the amplitude of thermal oscillation, $\omega / 2 \pi$ is the frequency and $\boldsymbol{K}=\sqrt{\omega / 2 k}$. The normalized subsurface temperature distribution ( $\left.\mathrm{T} / \mathrm{T}_{\mathrm{a}}\right)$ from Eqn. (2) is plotted against normalized distance, $\xi=x \sqrt{\omega / 2 k}$, in Figure 1(a) corresponding to various points of the thermal cycle. When the surface temperature is $T_{\max }(\omega t=0$, at the start of the cycle), the temperature decreases with distance and reaches $\mathrm{T}=\mathrm{T}_{\text {mean }}$ at $\xi \approx 1.6$. Then it gradually dies down approaching the $T_{\text {mean }}$ in the bulk. In the middle of the decreasing part of the thermal cycle $(\omega t=\pi / 2)$, even though the surface temperature is near $T_{\text {mean }}$, it can be seen that the subsurface temperature up to about $\xi=3$ is actually higher than $\mathrm{T}_{\text {mean }}$. This is because, although the external surface temperature has decreased, the excess heat below the surface has not yet dissipated, leading to a heat packet (inside which $\mathrm{T}>\mathrm{T}_{\text {mean }}$ ) formation in the subsurface region. With further surface cooling, say to about $\omega t=3 \pi / 4$, the peak temperature of the heat packet is seen shift to the inside of the solid-this is the transport of the heat-packet, even though the peak temperature of the packet itself is slowly diminishing with time. At this point of the cycle, the heat-packet region with $\mathrm{T}>\mathrm{T}_{\text {mean }}$ extends from about $\xi=1$ to about $\xi=4$ and represents a subsurface region that is actually hotter than $\mathrm{T}_{\text {mean }}$, but bound by relatively a cooler surface layer $(0<\xi<1)$ and the bulk $(4<\xi<\infty)$. The heat-packet diminishes rapidly when surface temperature is at the bottom of the thermal cycle. During the next heating phase of the cycle $(\omega t>\pi)$ the surface temperature increases again and sets up the conditions for the transport of heat-packet, again. Thus, a heatpacket is created in the subsurface region at $\mathrm{T}_{\max }$ and is transported into depth during cooling phase and dies down at some distance when $\mathrm{T} \sim \mathrm{T}_{\min }$. This process repeats with the repetition of 
surface temperature oscillation, repeatedly moving the heat packet region from the surface to the interior.

When the mean temperature of the thermal cycle coincides with the phase transition point (for example the transition from low temperature $\alpha$ phase to high temperature $\beta$ phase), the heat packet referred to above will actually be a traveling zone of $\beta$ phase, bounded by $\beta-\alpha$ boundary in the front and $\alpha-\beta$ boundary in the rear, which will repeatedly travel from the surface to the interior, upon repeated thermal cycling. Now, if some diffusing atoms are introduced at the surface, increased transport and supersaturation of these atoms in the subsurface regions might be possible, especially when the solubility of the diffusing atoms is large in $\beta$ phase, relative to that in $\alpha$ phase. With a proper temperature excursion and the periodicity of cycles, a relatively larger amount of solute can be transported to the interior by the moving zone of high temperature phase, which will lead to the accumulation of solute in the subsurface region. If this can be experimentally demonstrated, an increased transport of hardening species such as $\mathrm{C}, \mathrm{N}, \mathrm{O}, \mathrm{B}$ can be achieved in diffusion-based surface hardening processes of metals such as $\mathrm{Fe}, \mathrm{Ti}, \mathrm{Zr}, \mathrm{Co}$, leading to an increased non-equilibrium supersaturation of the hardening elements and producing increased hardness and/or the depth of the surface hardened layer. For brevity, this approach is termed here as, "cyclic-phase-change-diffusion (CPCD)."

We demonstrate here experimentally the above CPCD concept using titanium undergoing reversible $\alpha-\beta-\alpha$ phase transformations, by thermal cycling about the $\beta$-transus, and superimposed with B diffusion. Commercially pure titanium (CP-Ti, Grade 2) undergoes HCP $\alpha$ to $\mathrm{BCC} \beta$ phase transition upon heating across the phase transition temperature $\left(\sim 900^{\circ} \mathrm{C}\right)$. This phase transition is sensitive to oxygen as shown in the phase diagram (Figure 1b) drawn using the data of Jaffee et $\mathrm{al}^{9}$. There are actually two transus-temperatures ( $\alpha$-transus and $\beta$-transus) in Ti containing significant amount of oxygen, so, an effective thermal cycle must cross these boundaries. The solubility of $\mathrm{B}$ in $\beta$ is higher than that in $\alpha$ phase $^{10}$. Figure $1 \mathrm{~b}$ also shows the cyclic temperature limits $\left(880-920^{\circ} \mathrm{C}\right)$ used here with the mean temperature coinciding with the phase transition temperature, $900{ }^{\circ} \mathrm{C}$. When the specimen surface enters the $\beta$ phase field, a relatively large amount of $\mathrm{B}$ from the surrounding $\mathrm{B}$ medium will be dissolved in the subsurface 
layer. If this $\mathrm{B}$ is transported into the bulk by the traveling $\beta$-phase zone this will be later converted into elongated $\mathrm{TiB}$ phase (whiskers) when the subsurface region transforms to $\alpha$ phase field. The nature of formation of TiB whiskers and their structure during isothermal $\mathrm{B}$ diffusion are described in our previous works ${ }^{11},{ }^{12}$. The objective of this work is to show that using CPCD, increased B supersaturation in subsurface layer can be produced leading to a higher density of the hard boride $(\mathrm{TiB})$ whiskers and, hence an increase in the depth of hardening.

Thermal cycling diffusion and isothermal diffusion experiments were performed as follows. Samples of CP-Ti (Grade 2, composition in wt.\%: $0.07 \mathrm{Fe}, 0.12 \mathrm{O}, 0.007 \mathrm{C}, 0.006 \mathrm{~N}, 0.001 \mathrm{H}$ and bal. Ti) were metallographically polished. The samples were embedded in a sealed stainless steel crucible containing the diffusion pack with amorphous B (95-97\% pure) as the B source, along with an activator and a transport medium, as given in our previous work ${ }^{13},{ }^{14}$. The crucible was thermally cycled in a programmable furnace between $880-920^{\circ} \mathrm{C}$ for $2,5,9,13,17,20,34$ and 50 cycles, corresponding to total exposure times of 3, 6, 12, 18, 24, 27, 48 and $71 \mathrm{hrs}$. This temperature range was chosen to cross the $\alpha-(\alpha+\beta)$ and $(\alpha+\beta)-\beta$ phase boundaries at 0.12 wt. $\%$ $\mathrm{O}$ content (Figure 1b). Thermal cycles with 2 min. ramp-up and ramp-down times between 880 and $920^{\circ} \mathrm{C}$ were employed. The hold periods at the limit temperature were set at $0.5 \mathrm{hr}$ to allow $\mathrm{B}$ dissolution and $\mathrm{TiB}$ precipitation. For comparison, isothermal B diffusion experiments were performed at $850^{\circ} \mathrm{C}$ ( $\alpha$-phase field) and $1050^{\circ} \mathrm{C}$ ( $\beta$-phase field) for a total exposure time of 71 hrs. To assess the actual effectiveness of CPCD, isothermal diffusion experiments were also done at the "equivalent temperature" which is the cycle mean temperature $\left(900{ }^{\circ} \mathrm{C}\right)$. The temperature was controlled within $\pm 2{ }^{\circ} \mathrm{C}$, with a thermocouple directly in contact with the specimen. Boride layer thicknesses were measured, on metallographically polished and etched samples, at ten equally spaced locations on multiple micrographs to determine the average thicknesses and the standard deviations. In each measurement, the point of TiB whisker that is the farthest from the surface was taken as the boride layer thickness.

Table 1 presents a summary of the boride layer thicknesses obtained by CPCD as well as isothermal treatments. The average boride layer thicknesses obtained by CPCD are significantly higher than that obtained in any isothermal conditions at total exposure times $>12 \mathrm{hr}$. The layer thickness data are plotted in Figure 2 as a function of total treatment time. For the isothermal 
treatments performed at 850 and $1050{ }^{\circ} \mathrm{C}$, the TiB layer growth saturated in about 24 hours, with the layer thicknesses reaching about 30 and $55 \mu \mathrm{m}$, respectively. The isothermal diffusion at the equivalent temperature, i.e. at $900{ }^{\circ} \mathrm{C}$, also led to increased layer thicknesses, relative to the 850 and $1050{ }^{\circ} \mathrm{C}$ isothermal data, at all treatment times. A part of this data $(\leq 24 \mathrm{hr}$.) belongs to the previous work ${ }^{15}$ where it was shown that the enhanced boride layer growth is due to the anomalous and enhanced diffusivity of $\mathrm{B}$ in $\mathrm{Ti}$ at the phase transition temperature. Hence, any growth enhancement due to CPCD should be more than that resulting from the equivalent isothermal diffusion at $900{ }^{\circ} \mathrm{C}$. Indeed, it is remarkable to see in Figure 2 that the growth kinetics of the TiB layer is significantly enhanced by the CPCD- the average layer thickness approaches $125 \mu \mathrm{m}$ after 71 hours or 50 cycles with little evidence of saturation. The CPCD appears to be effective even at shorter times-for example; a layer thickness of about $55 \mu \mathrm{m}$ was obtained in 12 hours under cyclic conditions, whereas it would take about $>18$ hours at a higher isothermal diffusion condition $\left(1050{ }^{\circ} \mathrm{C}\right)$ to obtain a similar result. Further, the growth curve under cyclic conditions appears to shift toward a linear behavior, which may suggest that the boride layer growth may not be saturating even after a large number of thermal cycles.

Table 1. Average total boride layer thicknesses, along with standard deviations, obtained in cyclic-phase-change-diffusion and isothermal treatments.

\begin{tabular}{|c|c|c|c|c|c|c|c|c|}
\hline \multirow{2}{*}{$\begin{array}{c}\text { Time } \\
(\mathbf{h r} .)\end{array}$} & \multicolumn{2}{|c|}{$\begin{array}{c}\text { Isothermal } \\
\mathbf{8 5 0}\end{array}$} & \multicolumn{2}{c|}{$\begin{array}{c}\text { Equivalent } \\
\text { Isothermal } \\
\mathbf{9 0 0}\end{array}$} & \multicolumn{2}{c|}{$\begin{array}{c}\text { Isothermal } \\
\mathbf{1 0 5 0}{ }^{\circ} \mathbf{C}\end{array}$} & \multicolumn{2}{c|}{$\begin{array}{c}\text { Thermal Cycle } \\
\mathbf{9 2 0 - 8 8 0} \\
\mathbf{0 . 5} \text { hr. hold }\end{array}$} \\
\cline { 2 - 9 } & $\begin{array}{c}\text { Ave. } \\
(\boldsymbol{\mu m})\end{array}$ & $\begin{array}{c}\text { S.D. } \\
(\boldsymbol{\mu m})\end{array}$ & $\begin{array}{c}\text { Ave. } \\
(\boldsymbol{\mu m})\end{array}$ & $\begin{array}{c}\text { S.D. } \\
(\boldsymbol{\mu m})\end{array}$ & $\begin{array}{c}\text { Ave. } \\
(\boldsymbol{\mu m})\end{array}$ & $\begin{array}{c}\text { S.D. } \\
(\boldsymbol{\mu m})\end{array}$ & $\begin{array}{c}\text { Ave. } \\
(\boldsymbol{\mu m})\end{array}$ & $\begin{array}{c}\text { S.D. } \\
(\boldsymbol{\mu m})\end{array}$ \\
\hline 3 & 23 & 3.2 & 24 & 5 & 31 & 2.5 & 20 & 3 \\
\hline 6 & 24 & 3.5 & 39 & 8 & 37 & 3.4 & 36 & 6 \\
\hline 12 & 25 & 4.2 & 48 & 9 & 41 & 3.6 & 55 & 8 \\
\hline 18 & 26 & 5.4 & 55 & 12 & 50 & 5.7 & 57 & 9 \\
\hline 24 & 28 & 6.3 & 62 & 14 & 54 & 5.9 & 66 & 11 \\
\hline 27 & & & & & & & 79 & 15 \\
\hline 48 & 30 & 7.3 & 78 & 22 & 55 & 7 & 108 & 20 \\
\hline 71 & 31 & 7.5 & 101 & 28 & 55 & 7.2 & 126 & 23 \\
\hline
\end{tabular}

The SEM micrographs of boride layers obtained after 24, 48 and 71 hours of exposure during CPCD are shown in Figures 3(a)-(c), respectively. Generally, the boride layers consisted of a 
relatively thin $\mathrm{TiB}_{2}$ layer at the top (because of the proximity to B powder) and a much deeper $\mathrm{TiB}$ whisker layer growing into the depth. In the boride layer, between the long TiB whiskers, there are also continuous $\mathrm{Ti}$ regions in some places. Some of the TiB whiskers show serrated edges and some of them are partially dissolved due to the heavy etching used, which was necessary to properly reveal the boride layer. For comparison, the SEM micrographs of boride layers obtained after isothermal diffusion of boron at 850, 900 and $1050{ }^{\circ} \mathrm{C}$, for $71 \mathrm{hrs}$. are presented in Figure 4(a)-(c), respectively. After several trials an optimum etching solution was selected to reveal the pristine TiB whiskers-however, this seems to have partially anodized the titanium matrix, as indicated by the globular particles in the Ti matrix. It was not possible to avoid these artifacts and yet reveal the TiB structure clearly.

A comparison of the $\mathrm{TiB}$ layer structure (between figures 3(c) and 4(b)) reveals that the layers show relatively thicker, deeper and a high areal density of TiB whiskers in CPCD, compared to that obtained in the $900{ }^{\circ} \mathrm{C}$ equivalent isothermal condition. In the latter, the TiB whisker density is not as dense and uniform (Figure 4(b)) as it is in CPCD - the TiB whiskers are relatively thin and a much higher proportion of titanium is present between them. This indicates that CPCD leads to a higher supersaturation of B in the boride layer. According to Ti-B phase diagram, TiB is a line compound that is in equilibrium with $\mathrm{Ti}$, all the way up to its melting temperature, $\sim 2200^{\circ} \mathrm{C}$. Hence most of the $\mathrm{TiB}$ whiskers can be expected to have formed during the temperature excursions of the CPCD cycles. However, a small amount of TiB may form during cooling below beta-transus, because of the higher solubility of B in $\beta$-phase relative to that in $\alpha$ phase. In contrast, isothermal diffusion at $850{ }^{\circ} \mathrm{C}$ produced a relatively less deeper boride layer $(\sim 33 \mu \mathrm{m})$ with a lesser density of TiB whiskers (Figure $4(\mathrm{a}))$. The isothermal condition $1050^{\circ} \mathrm{C}$ produced a very different boride layer, with entire boride growth occurring in $\beta$-phase. Boride growth here leads to a relatively thick $\mathrm{TiB}_{2}$ layer and the underlying $\mathrm{TiB}$ layer with itself contains thick TiB whiskers (Figure 4(c)). This is due to the B-diffusion limitations in $\mathrm{TiB}_{2} / \mathrm{TiB}$ phases and is discussed in our previous work ${ }^{14,15}$. It appears that CPCD circumvents the diffusion limitation and leads to increased supersaturation of B and TiB formation, leading to a relatively deeper boride growth layers. The increased subsurface micro-hardness levels in CPCD, compared to the isothermal condtions, are illustrated in the supplementary document. 
In conclusion, the cyclic-phase-change-diffusion (CPCD) process has been demonstrated to produce a relatively deeper $(>125 \mu \mathrm{m})$ boride supersaturated layer in the subsurface region of $\mathrm{Ti}$ relative to that obtained by the isothermal $\mathrm{B}$ diffusion at a much higher temperature $\left(1050{ }^{\circ} \mathrm{C}\right)$ or at the equivalent isothermal condition $\left(900^{\circ} \mathrm{C}\right)$. More interestingly, the boride layer growth in CPCD did not appear to reach saturation. In contrast, all the isothermal treatments at temperatures far away (above and below the phase transition temperature) exhibited saturation of boride growth after $24 \mathrm{hrs}$. The CPCD process appears to produce a traveling beta phase region that transports $\mathrm{B}$ into interior where they eventually form $\mathrm{TiB}$ whiskers producing a more hardened boride layer with increased thickness, depth and area density of TiB phases.

\section{Acknowledgement}

National Science Foundation supported this research through the grant DMR-0737883 from Division of Materials Research and by the program on Designing Materials to Revolutionize our Engineering Future (DMREF) through the grant CMMI-1435758.

\section{Reference}

${ }^{1}$ R. Grange, Trans. ASM. 1 (1966) 26-48

${ }^{2}$ S. Jin, J. W. Morris, Jr. and V. F. Zackay, Metall. Trans. 6A (1975) 141-149

${ }^{3}$ K. Nakazawa, Y. Kawabe, S. Muneki, Mater. Sci. Eng. 33A (1978) 49-56

${ }^{4}$ S. Kohara, Metall. Trans. 7A (1976) 1239-1241

${ }^{5}$ O. A. Ruano, J. Wadsworth and O. D. Sherby, Metall. Trans. 13A (1982) 355-361

${ }^{6}$ D. C. Dunand and C. M. Bedell, Acta Mater. 44 (1996) 1063-1076

${ }^{7}$ V. Sista, P. Nash and S. S. Sahay, J. Mater. Sci. 49 (2007) 9112-9115

${ }^{8}$ H. S. Carslaw and J. C. Jaeger, Conduction of Heat in Solids, Oxford Science Publishers, Second Edition, Oxford, UK, 2008, p. 67

${ }^{9}$ R. I. Jaffee, H. R. Ogden and D. J. Maykuth, JOM 188 (1950) 1261-1266

${ }^{10}$ J. L. Murray, P. K. Liao and K. E. Spear, Bulletin of Alloy Phase Diagrams 7 (1989) 550-555

${ }^{11}$ S. S. Sahay, K. S. Ravichandran, R. Atri, B. Chen and J. Rubin, J. Mater. Res. 14 (1999) 42144223

${ }^{12}$ N. Tikekar, K. S. Ravi Chandran and A. Sanders, Scr. Mater. 57 (2007) 273-276 
${ }^{13}$ S. Aich and K. S. Ravi Chandran, Metall. and Mater. Trans. 33A (2002) 3489-3498

${ }^{14}$ B. Sarma and K.S. Ravi Chandran, Ceramic International 38 (2012) 6795-6805

${ }^{15}$ B. Sarma and K.S. Ravi Chandran, Acta Mater. 59 (2011) 4216-4228 


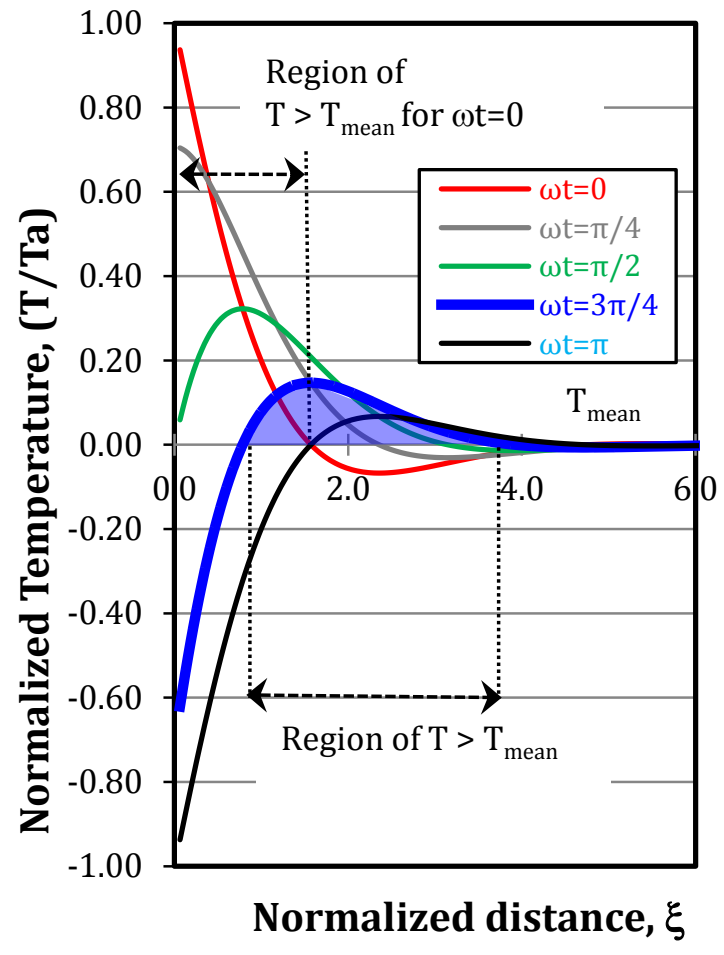

(a)

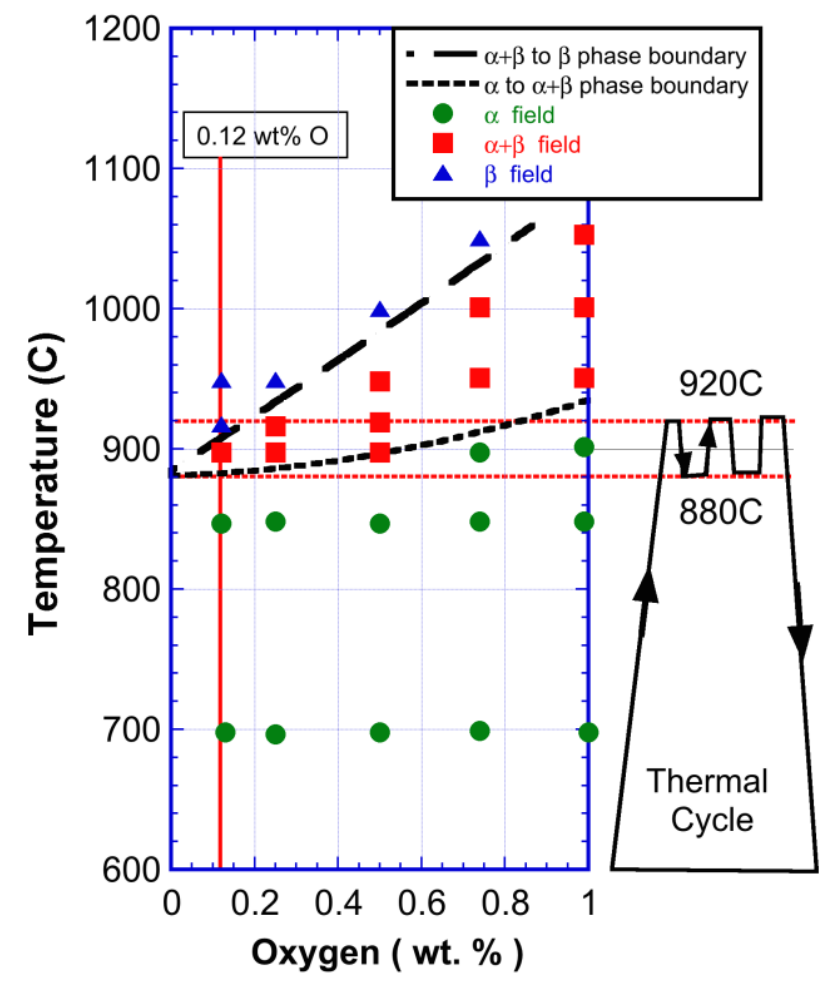

(b)

Figure 1. (a) Subsurface temperature distributions due to harmonic variation in surface temperature with the shaded area highlighting the travelling heat packet and (b) Ti-O phase diagram drawn using the data of Jaffee et al. [Ref.9] showing $\alpha, \beta$ and $\alpha+\beta$ phase fields, the bulk composition of $\mathrm{CP}-\mathrm{Ti}$, and the thermal cycle profile. 


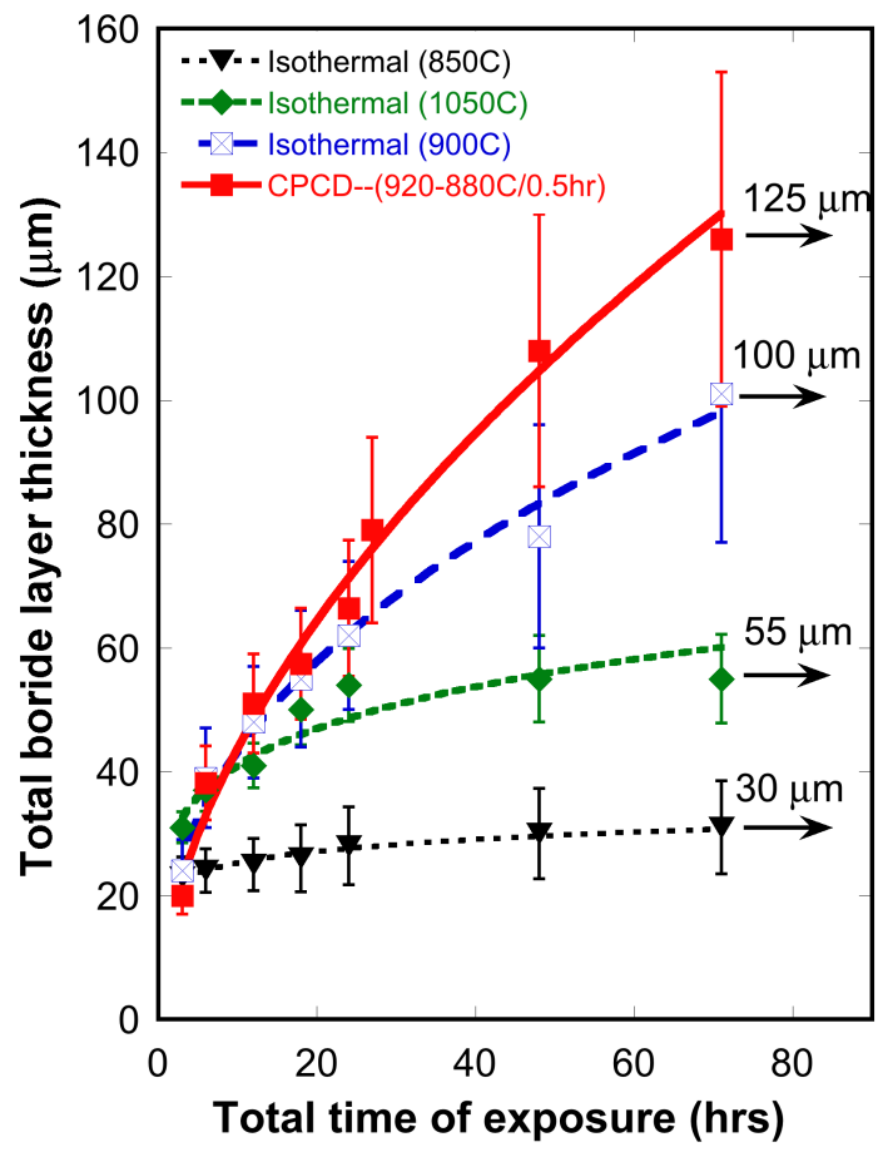

Figure 2. Total $\left(\mathrm{TiB}_{2}+\mathrm{TiB}\right)$ boride layer thicknesses obtained in CPCD process, as well as in isothermal diffusion treatments, plotted as a function of total exposure time 
$\mathrm{TiB}_{2}$ layer
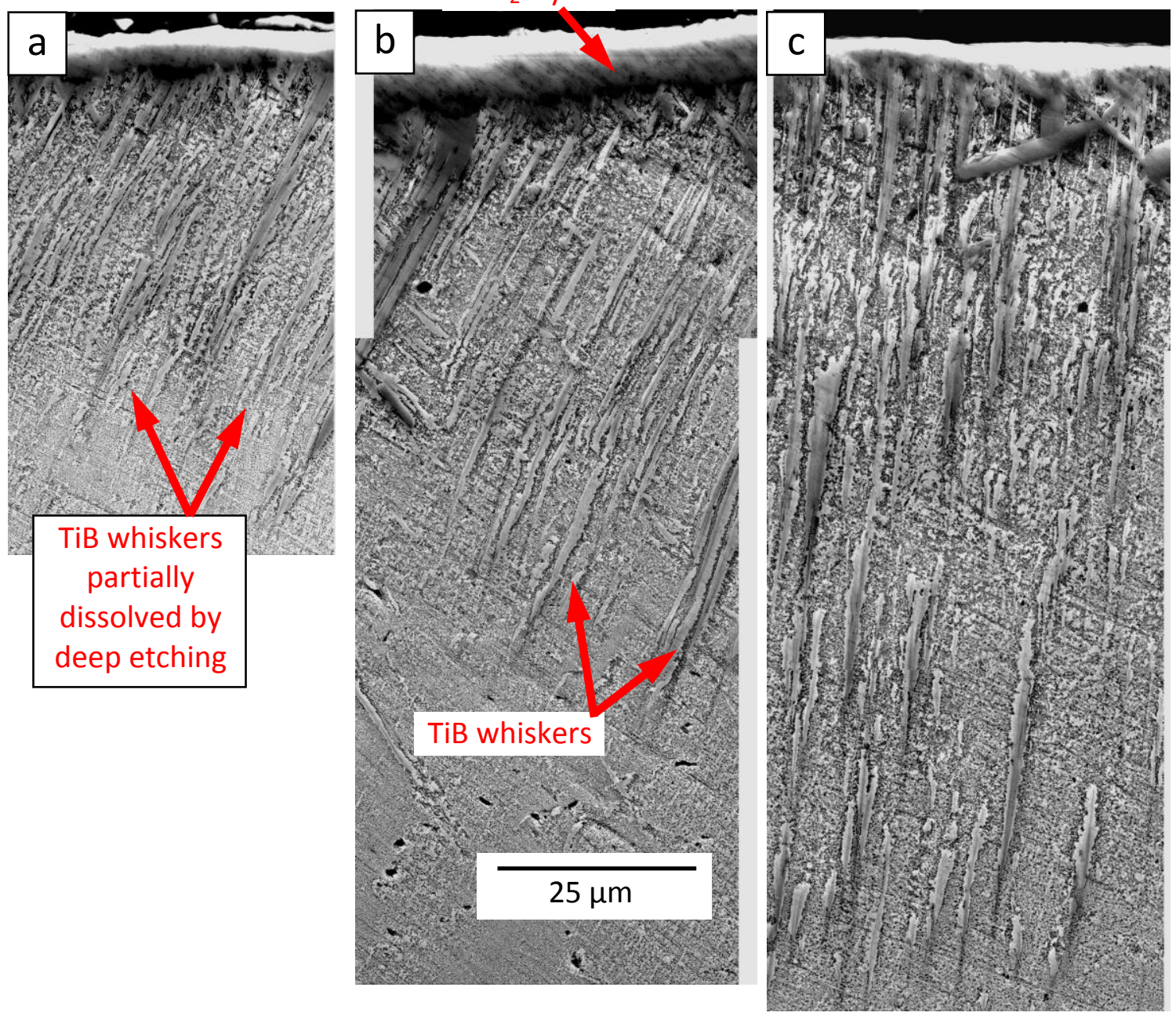

Figure 3. SEM micrographs of the boride layers produced under thermal cycling between 880$920{ }^{\circ} \mathrm{C}$ with a mean temperature of $900{ }^{\circ} \mathrm{C}$ for a total time of (a) $24 \mathrm{hrs}$. (17 cycles), (b) $48 \mathrm{hrs}$. (34 cycles), and (c) 71 hrs. (50 cycles). The specimens were deep etched to reveal the structure of $\mathrm{TiB}$ phase. 
$\mathrm{TiB}_{2}$ layer

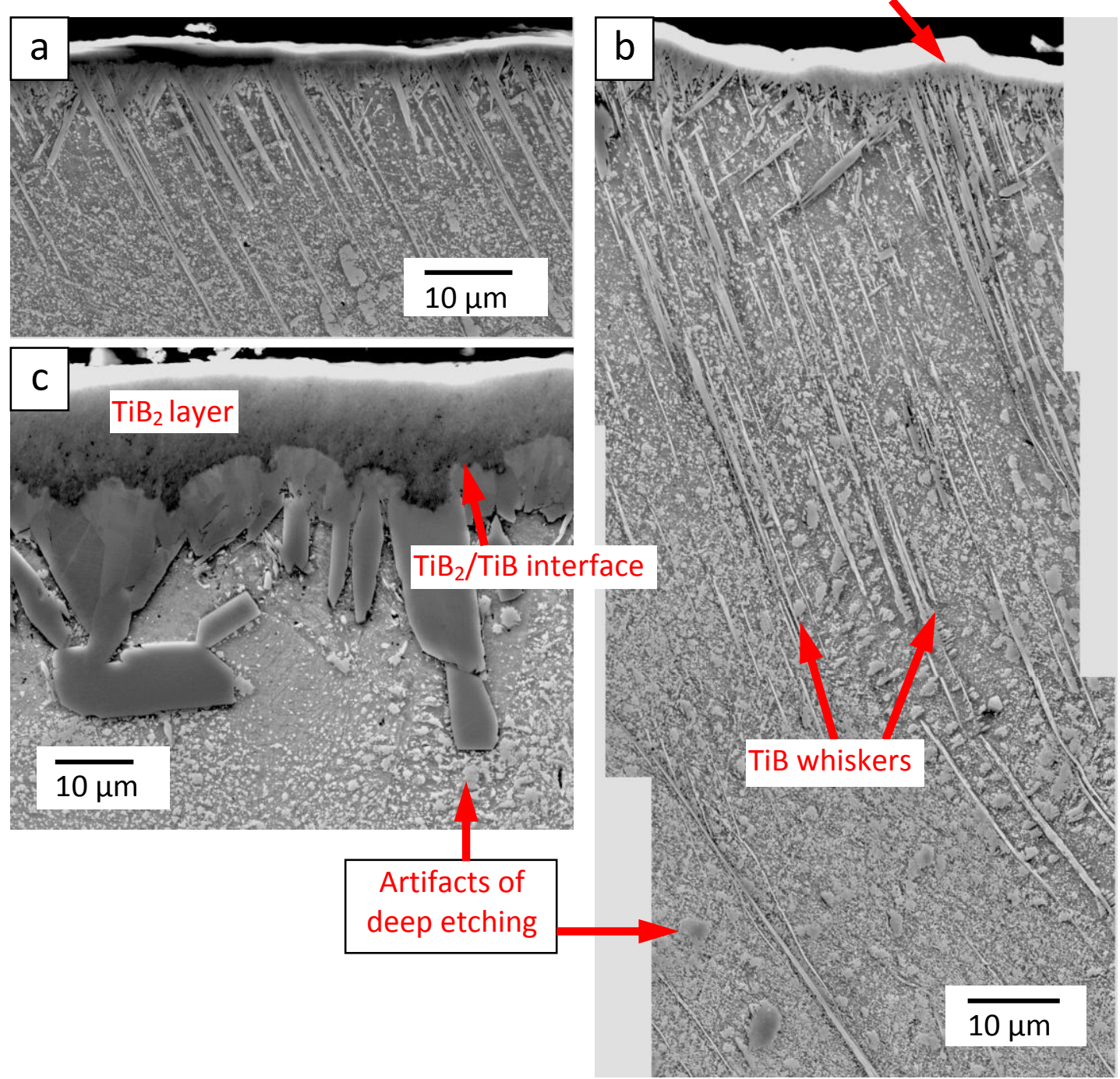

Figure 4. SEM micrographs of the boride layers produced under isothermal diffusion for 71 hours at (a) $850{ }^{\circ} \mathrm{C}$, (b) $900{ }^{\circ} \mathrm{C}$ and (c) $1050{ }^{\circ} \mathrm{C}$. The specimens were deep etched to reveal the structure of TiB phase. 


\section{Graphical Abstract (B. Sarma and K. S. Ravi Chandran)}

Title:

Superior surface hardening by cyclic-phase-change-diffusion (CPCD): Supersaturation of hard boride in titanium subsurface layer
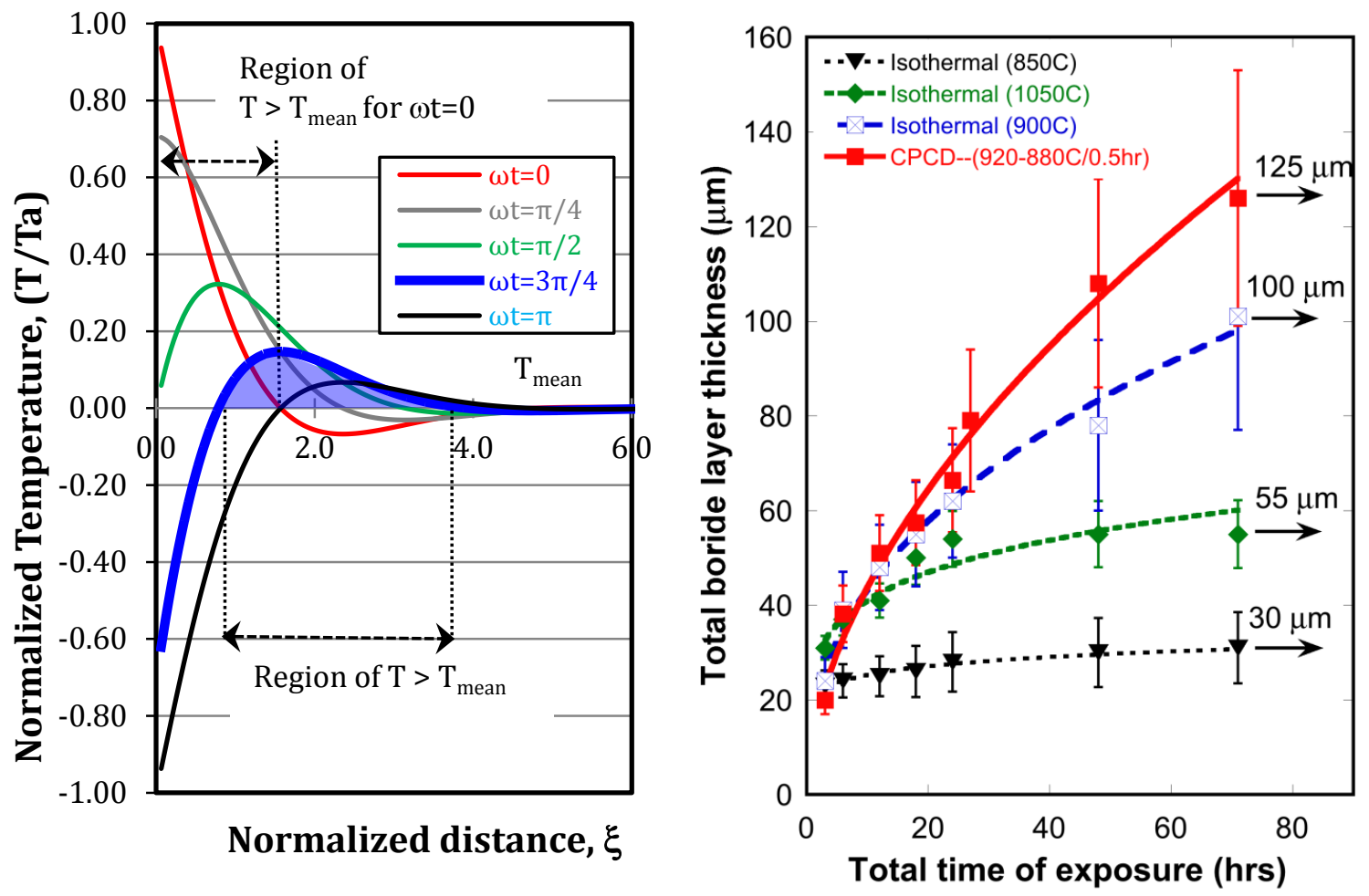